\section{Northwest Tribal Food Sovereignty Coalition: An intertribal collaboration}

\author{
Nora Frank-Buckner a * \\ WEAVE-NW project at the Northwest Tribal \\ Epidemiology Center
}

in collaboration with the Northwest Tribal Food

Sovereignty Coalition $\mathrm{b}$
Special JAFSCD Issue

Indigenous Food Sovereignty in North America

\section{Swette Center for
Sustainable Food Systems Arizona State University}

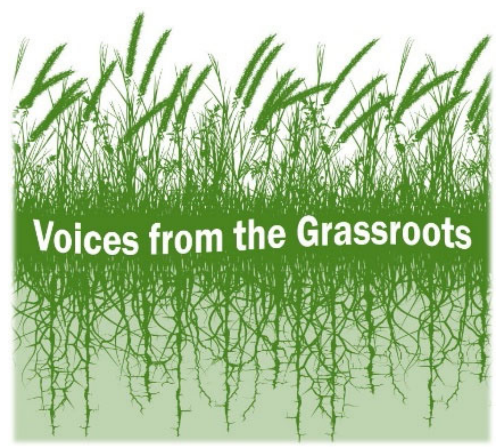

Submitted January 15, 2019/ Accepted January 15, 2019 / Published online October 15, 2019

Citation: Frank-Buckner, N., \& Northwest Tribal Food Sovereignty Coalition. (2019). Northwest Tribal

Food Sovereignty Coalition: An intertribal collaboration. Journal of Agriculture, Food Systems, and Community

Development, 9(Suppl. 2), 9-12. https://doi.org/10.5304/jafscd.2019.09B.001

Copyright (C) 2019 by the Authors. Published by the Lyson Center for Civic Agriculture and Food Systems. Open access under CC-BY license.

\begin{abstract}
American Indians and Alaska Native people experience large disparities in the prevalence of preventable, diet-related diseases directly associated with the lack of access to healthy, traditional food. The Northwest Tribal Food Sovereignty Coalition, a tribal-driven network, is an opportunity for tribes, tribal organizations, and allied partners to organize efforts that are driven by cultural revitalization, community empowerment, and the use of innovative strategies to improve the health of the people and reclaim food sovereignty. This reflective essay aims to discuss the process of the development,

a * Corresponding author: Nora Frank-Buckner, MPH, WEAVENW Project Coordinator, 2121 SW Broadway, Suite 300; Portland, Oregon 97201 USA; +1-503-416-3253; nfrank@npaihb.org

b The Northwest Tribal Food Sovereignty Coalition is a tribaldriven network encompassing 27 tribes, 17 external organizations, and three working groups. The NTFSC is facilitated and coordinated by the WEAVE-NW (Wellness for Every American Indian to View and Achieve Health Equity) project.
\end{abstract}

recruitment, and activities of this newly formed coalition.

\section{Keywords}

Tribal Food Sovereignty, Food Systems, Coalitions, Traditional Foods, American Indian/Alaska Native Nutrition

\section{Introduction}

There is an epidemic of preventable, diet-related diseases directly associated with the lack of access to healthy, traditional foods, and medicines. American Indian and Alaska Native people (AI/AN) experience higher rates of chronic diseases, such as type II diabetes and cardiovascular disease, compared to other American populations (Indian Health Service, 2018). The cause of this is complex and multifaceted. It is rooted in colonization and the removal of tribal people from their homelands, often to areas that are less rich in natural resources. Federal policies throughout history have affected the way AI/AN people interact with the food 
system. Prior to colonization, AI/ANs obtained their food through hunting, fishing, and gathering. In the Northwest, traditional AI/AN foods consist of a diverse range of plants and animals such as wild berries, roots, wild greens, salmon, shellfish, elk, and deer, and clean water from streams rich in minerals. The traditional native foods are rich in nutrients and free of the unhealthy preservatives and additives common in highly processed foods, which are often the only choices available at small grocery stores and markets near tribal communities.

For decades, tribes in the Northwest region (Idaho, Oregon, and Washington) have implemented food sovereignty and traditional food projects to reclaim their traditional food knowledge and traditional food system to improve the health of their communities. The Northwest Tribal Food Sovereignty Coalition (NTFSC) provides an opportunity to organize these efforts to achieve food sovereignty and optimal health. Food sovereignty can be defined as the right of indigenous nations to define their own diets and shape food systems that are harmonious with their spiritual and cultural beliefs, knowledge, and values (Well for Culture, 2018). Food sovereignty is about tribal communities providing food to their members through culture, traditions, policies and/or law (local, state, or federal), and economic development (Echo Hawk Consulting, 2015).

The NTFSC is facilitated and coordinated by the WEAVE-NW (Wellness for Every American Indian to View and Achieve Health Equity) project at the Northwest Tribal Epidemiology Center (NWTEC) and is funded through a cooperative agreement with the Centers for Disease Control and Prevention (CDC), under the Good Health and Wellness in Indian Country initiative (CDC, 2016). The NWTEC is one of 12 national Tribal Epidemiology Centers and serves the 43 federally recognized tribes in Idaho, Oregon, and Washington. NWTEC is responsible for collecting tribal health status data, conducting evaluations, doing epidemiologic surveillance, and assisting tribes in identifying local priorities for healthcare delivery and health education programs (Northwest Portland Area Indian Health Board [NPAIHB], n.d.-a).
From 2015 to 2017, WEAVE-NW provided 14 subawards to Northwest tribes. Many of these tribes focused on the development or strengthening of community gardens, traditional food programs, food policy, partnerships, sustainability, and food sovereignty assessments. With shared aims of food system change approaches, the tribes requested more opportunity for intertribal collaboration to share resources and tools.

Following the 2016 Native American Nutrition conference in Prior Lake, Minnesota, WEAVENW staff and tribal subawardees debriefed following the breakout sessions and discussed what would be useful moving forward. These early conversations led to the creation of the NTFSC, with additional support being gathered from community leaders, youth, elders, and tribal employees at other meetings, trainings, and events in the Northwest region. One tribal member expressed, "There are so many tribes that have developed curriculum and resources [around traditional foods], it would be nice to share these with each other so we don't have to recreate the wheel."

WEAVE-NW serves as the backbone organization for the facilitation and coordination of the NTFSC. Forming the coalition currently are 27 tribes, 17 external organizations, and three working groups. The members come from a wide range of professions and backgrounds, including elders, tribal leaders, garden coordinators, traditional food educators, health professionals, and extension agents. Each of these members brings regional- or community-level expertise, leadership, and skill sets relevant to the region.

In the early stages of development, a strategic action planning meeting was held. The NTFSC identified two areas of focus for their first year: (1) to develop a media campaign on the importance of traditional foods (see the logo, Figure 1), and (2) to host a regional gathering for a cultural and knowledge sharing of traditional foods, medicine, and culture. These two focus areas served as a starting point for two of the three working groups (the media and gathering workgroups). Tribal and organizational partners self-selected into each working group and began making an action plan to carry out these activities. 
Figure 1. Northwest Tribal Food Sovereignty Coalition Logo Representing the Diversity of Plant and Wildlife of the Region

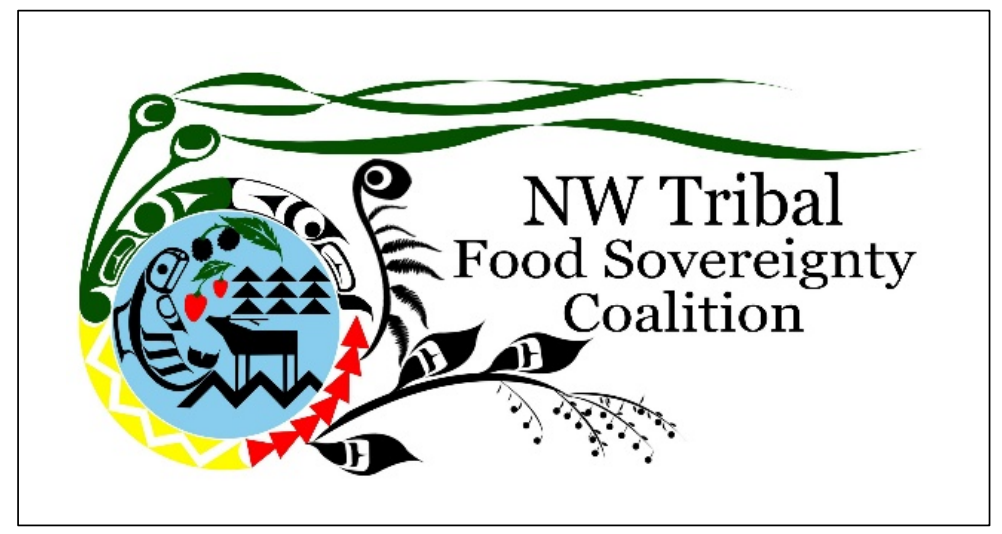

plans to hire a design team to help the Media Workgroup move forward with the planning and implementation of a marketing strategy.

One of the main highlights of this first year was the planning and implementation of the NTFSC's gathering. The gathering was held in fall 2018 on the Port Madison Indian Reservation of the Suquamish Tribe. Over 160 participants from across the region attended this event, including youth, elders, community leaders, tribal leaders, tribal and nontribal organizational partners, and tribal staff.

After the initial meeting, a leadership workgroup met via conference call and in person to brainstorm the vision, mission, and values of the coalition. Although still in development, the NTFSC's working mission is to "reclaim our indigenous knowledge to maintain and improve our health and quality of life for ourselves and future generations." A final version of these will be published on the NPAIHB website under the NTFSC page (NPAIHB, n.d.-b).

The Media Workgroup has participated in an initial survey to brainstorm ideas for a target audience and main message. There is more work to be done to narrow down these ideas. WEAVE-NW

\section{Figure 2. Plated Food from the "Native Chopped" Cooking Competition Awaiting Critique by the Elder Judges}

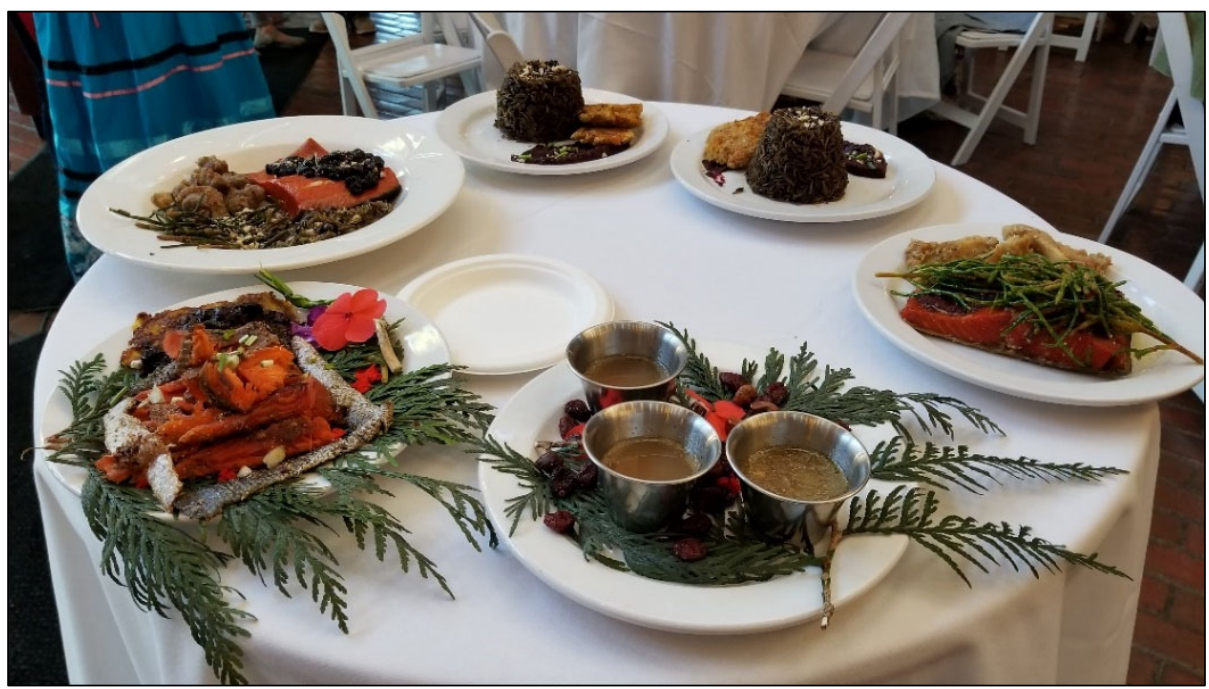

It was important to the Gathering Workgroup to include presentations on community-level projects addressing food sovereignty. Specifically, the chairman of the NPAIHB, Andy Joseph (of The Confederated Tribes of Colville), was invited to give a keynote presentation. The chairman presented on his tribe's First Foods, including information on the restoration of salmon runs, the recovery of lamprey, and the reintroduction of wildlife. In addition, the workgroup invited three additional communities to present on their work in the form of a panel. The agenda also included a traditional foods meet-and-greet (where participants showcased various traditional foods and medicines), skill-building break-out sessions, a "Native Chopped" cooking competition (Figure 2), and a traditional dinner with salmon, shellfish, and other traditional foods (Figure 3).

Both formal and anecdotal evaluation of the gathering demonstrated that there was an overwhelmingly positive response to the event. One participant said, "I feel so in my native element! After eating all of these traditional 
foods, I can feel it pumping through my veins!"

Another participant wrote, "[The biggest takeaway was]... policy and how it needs urgent attention. I needed the recharge with this gathering to continue the work at home."

The feedback indicated that participants most liked having the opportunity to connect, learn from one another, and share resources, as well as having traditional foods incorporated into all the meals.

In the chairman's notes in NPAIHB's fall 2018 newsletter, Mr. Joseph states, "The gathering highlighted communities across the Northwest that are working on food sovereignty and food system change. Our traditional foods and medicines are healing, and they can help us prevent chronic diseases."

The momentum and energy from the gathering continued, and there were requests for a Food Sovereignty Assessment Tool training. WEAVENW partnered with Valerie Segrest of Muckleshoot Tribe and the First Nations Development Institute (FNDI) to provide a day-and-a-half training on the Food Sovereignty Assessment Tool in late fall 2018 (FNDI, 2014). Many Northwest tribes expressed interest in conducting assessments in their own communities and developing action plans.

It is through opportunities like the NTFSC gathering, trainings, and meetings that members are able to work intertribally and across sectors to address issues facing the tribal food system. Continued development, recruitment, and sustainability planning of the NTFSC are necessary to ensure all

\section{Figure 3. Salmon That Would be Served at the Traditional Dinner for the Gathering Cooking over the Fire}

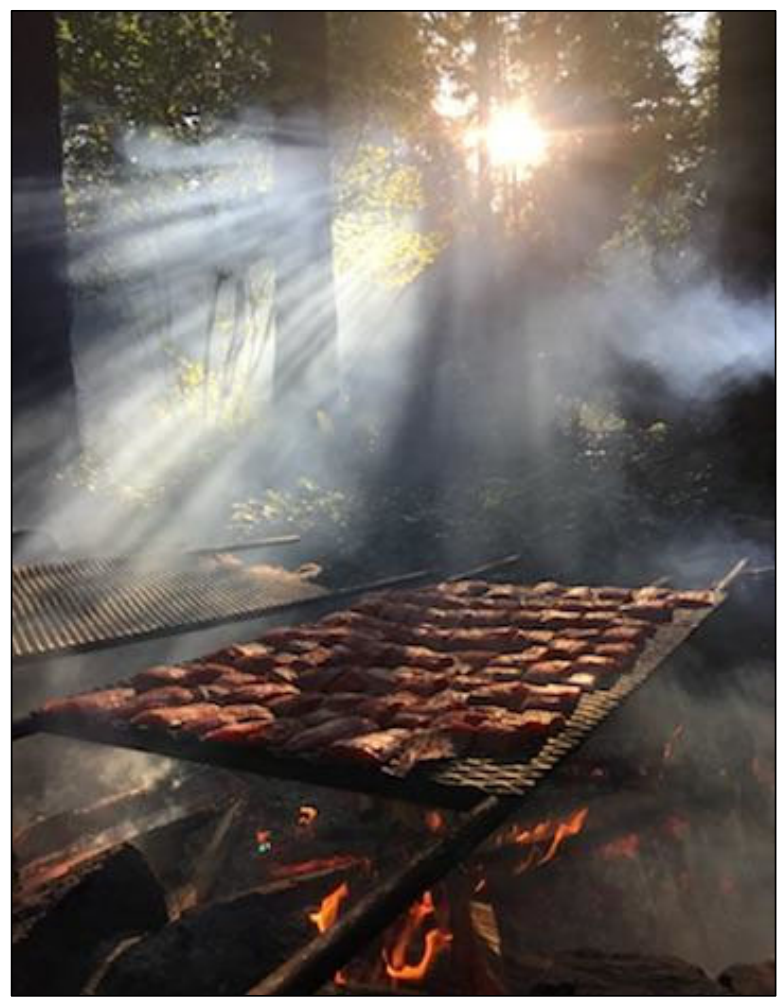

Northwest tribes are heard and are recognized for their leadership in the food sovereignty movement.

Together, we are building a strong collaboration of tribal nations, tribal organizations, and allied partners to work effectively toward true tribal food sovereignty.

\section{References}

Centers for Disease Control and Prevention (CDC). (2016, March 6). Good Health and Wellness in Indian Country.

Retrieved from the National Center for Disease Prevention and Health Promotion website:

https://www.cdc.gov/chronicdisease/resources/publications/aag/indian-country.htm

Echo Hawk Consulting. (2015). Feeding ourselves: Food access, health disparities, and the pathways to healthy Native American communities. Longmont, CO: Echo Hawk Consulting.

First Nations Development Institute. (2014). Food Sovereignty Assessment Tool, $2^{\text {nd }}$ Ed. Longmont, CO: First Nations Development Institute. Retrieved from https://www.firstnations.org/publications/food-sovereignty-assessment-tool-2nd-edition/

Indian Health Service. (2018, April). Disparities. Retrieved from https://www.ihs.gov/newsroom/index.cfm/factsheets/disparities/

Northwest Portland Area Indian Health Board [NPAIHB]. (n.d.-a). EpiCenter. Retrieved June 12, 2019, from http://www.npaihb.org/epicenter

NPAIHB. (n.d.-b). NW Tribal Food Sovereignty Coalition. Retrieved June 12, 2019, from http://www.npaihb.org/ntfs-coalition/

Well for Culture. (2018). Tribal food sovereignty. http://www.wellforculture.com/tribal-food-sovereignty/ 